\title{
The Flow Stress Constitutive Equation and Its Simulation of the Electro Plastic Effect
}

\author{
Shuhua Peng and Yao $\mathrm{Li}^{*}$ \\ School of Electrical-mechanical Engineering, Jianghan University, Wuhan, 430056, China \\ ${ }^{*}$ Corresponding author
}

\begin{abstract}
The tensile experiments were carried out under the condition of different pulse current on pure tantalum. A synthetic plasticity constitutive form $\mathrm{H}-\mathrm{V}$ model describing the flow stress as a function of strain under the influence of pulse current was developed to fit the measured data -flow stress and strain curve. The Hollomon, Voce and $\mathrm{H}-\mathrm{V}$ models were fit for pure tantalum using a series of tensile tests up to the uniform strain conducted over a range of pulse current. The three models then were to predict the behavior of the tests outside the range. The results show that the $\mathrm{H}-\mathrm{V}$ model provides more accurate predictions of strain behavior than the other two during the tensile with pulse current. The fitted value $\mathrm{R} 2$ of the model is 0.99 and the mean error is $6.5 \%$.
\end{abstract}

Keywords-electroplastic effect; Pulse current; Constitutive equations; Work hardening; Strain; stress

\section{INTRODUCTION}

Electro plastic effect refers that metal appears to be of increased in plasticity and decreased in resistance in the process of plastic deformation by applying pulse current. Some earlier researches had also suggested that pulse current could improve the plastic deformation. But the the function relationships among the strain, stress and pulse current are still in their explore. The relationships could reflect the dependencies between the flow stress and the strain under the condition of electricity. In order to establish a reliable strain-stress equation, this study analyzed the measured flow stress-strain and the rheological model was described in the range of stress,strain and pulse current based on pure aluminum and pure titanium specimens in plastic deformation.

\section{The Establishment AND ANALYSIS OF FlOW StRESS CONSTITUTIVE EQUATION}

\section{A. The Establishment of Flow Stress Constitutive Equation}

The plasticity constitutive equation describing the flow stress as a function of strain, strain-rate, temperature and other related influencing factors has been scratching our heads because of the range, diversity and complexity of the relationship between the factors and functions. But it is well known that after deformation, a series of changes occur in metallic internal organization, which will leads to the change of metal properties especially mechanical properties. With the increase of deformation extent, the phenomenon called workhardening in which strength increases and the plasticity decreases can not be ignored. Its function makes the most contribution to the constitutive equation [2]
In addition to integrate related influencing factors and their functions, there are myriad ways to choose and combine in which we prefer to incorporate these factors and functions via a linear combination [3].A new constitutive equation is proposed as the four multiplicative functions, showing as (1):

$$
\sigma=\sigma(\varepsilon, \dot{\varepsilon}, J, T)=\phi(\varepsilon) \cdot \mathrm{f}(\dot{\varepsilon}) \cdot \mathrm{g}(J) \cdot \mathrm{h}(T)
$$

where $\sigma$ is flow stress, $\varepsilon$ is strain, $\dot{\varepsilon}$ is strain-rate, $\mathrm{T}$ is temperature, $\mathrm{J}$ is pulse current, functions $\varphi, \mathrm{f}, \mathrm{g}$ and h represent the effect of work-hardening and other corresponding factors.

Recent work is conducted to integrate the constitutive equations relating flow stress with strain-rate, strain and temperature and has made some progress, such as integrated constitutive equations of strain and stress: Brown-Anand model, MTS model, thermal softening functions[4-6]: Linear model, Power law model, strain hardening functions: Hollomon model[7] and Voce model[8]. The existence of effect to the strain hardening function by application of the current pulse field in the deformation process is not allowed to ignore, so we integrate the influencing factor pulse current and its functions via a linear combination to combine the Hollomon model and Voce model.

$$
\begin{array}{ll}
\text { Hollomon model } & \sigma_{H}=A \varepsilon^{\mathrm{n}} \\
\text { Voce model } & \sigma_{V}=B\left(1-C \mathrm{e}^{\mathrm{m} \varepsilon}\right) \\
\text { Connection model } & \chi(J)=\left[\chi_{1}-\chi_{2}\left(J-J_{0}\right)\right] \\
H-V \text { synthesis equation } & \sigma(\varepsilon, J)_{H V}=\chi(J) \sigma_{H}+[1-\chi(J)] \cdot \sigma_{V}
\end{array}
$$

where $\mathrm{J}_{0}$ is reference pulse current (general is 0 , which means the specimen is not subjected to pulse current ), A, B, C, m, n are material constants. The connection model can control the whether the stress constitutive equation is more like $\mathrm{H}$ model or $\mathrm{V}$ model at the condition of high pulse current density depending on the value of . When $\chi(J)=1$, the $\mathrm{H}-\mathrm{V}$ model becomes a pure $\mathrm{H}$ model and when $\chi(J)=0$, it becomes a pure $\mathrm{V}$ model.

The influence of electro plastic effect on stress is not only related to the pulse current but also takes the pre-plastic strain into account 


$$
\sigma_{\mathrm{n}}=\mathrm{f}\left(\varepsilon_{\mathrm{n}}\right)-\sum_{\mathrm{m}=1}^{\mathrm{n}} \phi\left(\varepsilon_{\mathrm{m}}\right) \psi^{\prime} \mathrm{q}_{\mathrm{m}-1} \Delta \mathrm{q}_{\mathrm{m}}
$$

The appropriate equation which express the influence of the pulse current on stress and strain can be represented in a form of (2)[9] :

$$
\sigma(J)=C_{0}\left[1+C_{1} \varepsilon-C_{2}\left(\frac{\mathrm{q}_{0}}{\mathrm{q}}\right)^{3} \cdot \exp \left(3 \frac{\mathrm{q}_{0}}{\mathrm{q}}\right) \cdot \varepsilon\right]
$$

where $\sigma_{0}$ is initial yield stress, $\varepsilon$ is strain, $\mathrm{q}_{0}$ is the effective pulse current, $\mathrm{C}_{0}, \mathrm{C}_{1}, \mathrm{C}_{2}$ are constants.

According to Linear thermal softening function [10] $\mathrm{h}(T)=\sigma_{\text {iso }}\left(1-\beta\left(T-T_{\mathrm{r}}\right)\right)$ and Power law strain rate sensitivity functions [11] $\sigma=\sigma \dot{\varepsilon}_{0}\left(\frac{\dot{\varepsilon}}{\dot{\varepsilon}}\right)^{\mathrm{m}}$, for the same strain rate and temperature, their influence to flow stress is certain. During the tensile deformation of tantalum specimens, the tensile tests is perform at a constant speed of $1.67 \times 10^{-3} \mathrm{~s}^{-1}$, and electric fan is employed to decrease the temperature of the sample, so the pulse current heating effect can be ignored. Combine the (1) (2) (3), we can get a new one:

$$
\sigma=\sigma(\varepsilon, \dot{\varepsilon}, J, T)=\phi(\varepsilon, J) \cdot \mathrm{g}(J)=\sigma(\varepsilon, J)_{H V} \cdot \sigma(J)
$$

Apply function $\sigma(\varepsilon, J)_{H V}$ and $\sigma(J)$ to Equation(4):

$$
\sigma=\chi(J) \sigma_{H}+[1-\chi(J)] \cdot C_{0}\left[1+C_{1} \varepsilon-C_{2}\left(\frac{\mathrm{q}_{0}}{\mathrm{q}}\right)^{3} \cdot \exp \left(3 \frac{\mathrm{q}_{0}}{\mathrm{q}}\right) \cdot \varepsilon\right]
$$

After sorting and reduction, the flow stress constitutive equation of the electro plastic effect on pure tantalum can be represented as:

$$
\sigma=\sigma(\varepsilon, J) \cdot g(J)=\left[\chi(J)\left(A \varepsilon^{n}\right)+(1-\chi(J)) \cdot B\left(1-C^{-m}\right)\right] \cdot\left[H+C_{1} \varepsilon+C_{C}\left(\frac{\mathrm{q}_{0}}{\mathrm{q}}\right)^{3} \cdot \exp \left(3 \frac{\mathrm{q}_{0}}{\mathrm{q}}\right) \cdot \varepsilon\right](5)
$$

\section{B. The Analysis of Flow Stress Constitutive Equation}

In order to simulate the flow stress constitutive equation of the electro plastic effect on pure tantalum reasonably and effectively, we use the least square method, then select a high and low value of each variable as initial values, at last 29 sets of staring parameters is produced. The approximate ranges of the initial values are chosen on the basis of practical experience and material constant. If the initial values are out of the range or inadequacy, new initial values are chose to match the equation of that condition. The fitting will not end until the best match coefficients are chosen for each variable when error margin of plus or minus between the absolute value and the standardized residuals is less than 0.01. In the same way, When $\chi(J)=1$ and $\chi(J)=0$, the best match coefficients can be chosen foe each $\mathrm{H}-\mathrm{V}$ model. The best match values are showed in Table 1 .

TABLE I. BEST MATCH VALUES

\begin{tabular}{|c|c|c|c|}
\hline variable & $\begin{array}{c}\text { H-V model Best } \\
\text { match }\end{array}$ & $\begin{array}{c}\text { H model Best } \\
\text { match }\end{array}$ & V model Best match \\
\hline$\chi(\mathrm{J})$ & 0.9250 & 1 & 0 \\
\hline $\mathrm{A}$ & -3.0045 & 4.0042 & - \\
\hline $\mathrm{n}$ & 5.0377 & -1.0300 & 6.3282 \\
\hline $\mathrm{B}$ & 11.9271 & - & 0.7763 \\
\hline $\mathrm{C}$ & 0.6135 & - & 5.8816 \\
\hline $\mathrm{m}$ & 12.9126 & - & 83.8617 \\
\hline $\mathrm{H}$ & 360.2118 & 1.5457 & 15.8679 \\
\hline $\mathrm{C}_{1}$ & 160.0251 & 55.0064 & 31.6595 \\
\hline $\mathrm{C}_{2}$ & 51.7987 & 118.2752 & \\
\hline
\end{tabular}

The reliability of the flow stress constitutive equation of the electro plastic effect on pure tantalum can be validated by the experimental flow stress and strain data on the condition different pulse current. Using the Matlab, the flow stress model can be matched the true stress and strain data. The comparison between material's the real stress, strain and model matching curve, under different pulse current conditions, are shown in figure 1,2 and 3. As shown in figures, data points are the experimental flow stress and strain under the condition of different pulse current, and the curves are the flow stress based on corresponding flow stress constitutive equation matched strain. The process of experimental strain-stress curve measuring, we apply the dynamic strain indicator to displace changes of the true stress-strain curve and then data points in the curve are exported by excel. So the specimen's real stress under the conditions of different pulse current in the process of tensile deformation can be gotten. In the figure 1 , at the condition of strain rate of $\mathbf{1 . 6 7 \times 1 0 ^ { - 3 }} \boldsymbol{s}$, according to $\mathrm{H}-\mathrm{V}$ model, the simulate curve was consistent with the date points by experiment. The second place of the simulation effect is the $\mathrm{V}$ model (as figure 2) and $\mathrm{H}$ model is worst (as figure 3 ). The fitting coefficient R2 of the trend line determined by $\mathrm{H}-\mathrm{V}$ is 0.999 , V model of R2 is 0.96409 and $\mathrm{H}$ model is 0.88368 . When the fitting coefficient R2 is closer to1, the degree of the matching is higher.

According to the stress strain curve of different model as shown in figures, at the first when strain is small, the differences of three modes are small and the three models' simulation prediction is accurate compared with the experimental values. But the difference is more and more obvious when the strain increases. The flow stress constitutive equation of $\mathrm{H}-\mathrm{V}$ model's prediction is more accurate than Hollomon and Voce model after plastic deformation. Error analysis results of $\mathrm{H}-\mathrm{V}$ model show that the maximum error between the experimental flow stress and the simulative value is $7.9 \%$, and the average error is $6.5 \%$. It shows that the flow 
stress constitutive equation model based on the influence of pulse current has high calculation precision, and can be able to meet the needs of the engineering application. Finally simulation results show that root mean square error of the $\mathrm{H}-\mathrm{V}$, $\mathrm{V}, \mathrm{H}$ model is respectively $2.42,26.32,39.26$. Therefore, the flow stress constitutive equation of the electro plastic effect on pure tantalum, proposed by this paper can describe the pure tantalum's flowing behavior well in the process of the plastic deformation with pulse current.

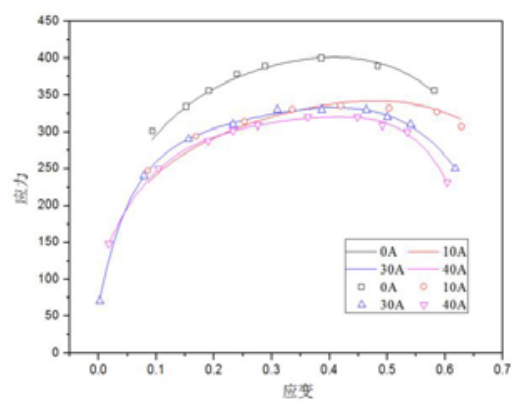

FIGURE I. THE SIMULATION RESULT OF H-V MODEL

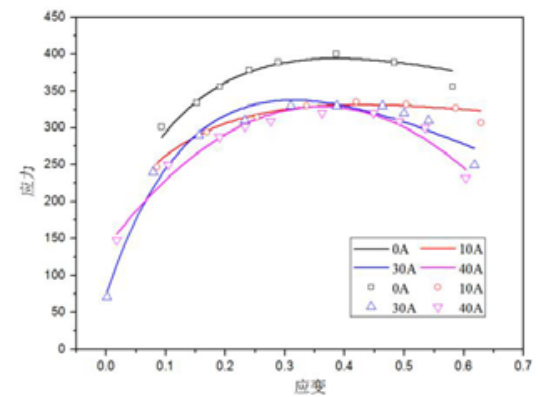

FIGURE II. THE SIMULATION RESULT OF V MODEL

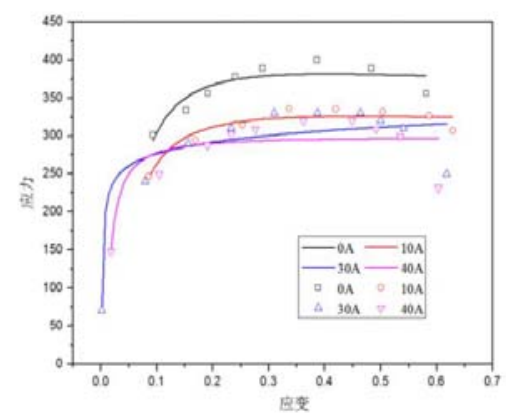

FIGURE III. THE SIMULATION RESULT OF H MODEL

\section{CONCLUSION}

A synthetic plasticity constitutive form $\mathrm{H}-\mathrm{V}$ model describing the flow stress as a function of strain under the influence of pulse current was proposed on the basis of Hollomom model and Voce model to fit the measured dataflow stress and strain curve. The simulation results show that the fitting coefficient R2 of the trend line determined by $\mathrm{H}-\mathrm{V}$ model is 0.999 , Voce model is 0.96409 at the second place, and the last is Hollomon model 0.88368. Root mean square error of the $\mathrm{H}-\mathrm{V}, \mathrm{V}, \mathrm{H}$ model is respectively $2.42,26.32,39.26$. The results show that $\mathrm{H}-\mathrm{V}$ Model fit to the uniform stress-strain curve, provides more accurate predictions at large stress-strain behavior in the process of electro plastic effect than $\mathrm{H}$ and $\mathrm{V}$ models in the literature.

Bered footnote on the first page.

\section{REFERENCES}

[1] Wu Quanxing, "The Application of Tantalum and its alloy," J. Rare Metals Letters, vol. 35, pp. 23-24, 2002.

[2] Ji Hyun Sung, Ji Hoon Kim and R H Wagoner, "A plastic constitutive equation incorporating strain, strain-rate and temperature," J. International Journal of Plasticity, vol. 26, pp. 1746-1771, 2010.

[3] Lin M R and Wagoner R.H, "Effect of temperature, strain, and strain rate on the tensile flow stress of I. F. steel and stainless steel type 310," J. Scripta Metallurgica, vol. 20, pp. 143-148, 1986.

[4] Laasraoui A and Jonas J J, "Prediction of steel flow stresses at high temperature and strain rates," J. Metallurgical Transaction A, vol. 22A, pp. 1545-1558, 1991 .

[5] Wu Wenxiang, Han Yi and Zhong Hao, "Flow stress behavior of 2026 aluminum alloy under hot compression deformation," J. The Chinese Journal of Nonferrous Metals, vol. 19, pp. 1403-1408, 2009.

[6] Li Xuesong, Chen Jun and Zhang Hongbing, "Constitutive model for hot deformation of 6082 aluminum alloy," J. The Chinese Journal of Nonferrous Metals, vol. 18, pp. 1769-1774, 2008.

[7] Koc P and Stok B, "Computer aided identification of the yield curve of a sheet metal after onset of necking," J. Computational Materials Science, vol. 31, pp. 155-168, 2004.

[8] Kleemola H J and Niemenen M A, "On the strain hardening parameters of Metals," J. Metallurgical and Materials Translations B, vol. 5, pp. 1863-1866, 1974.

[9] Georgy A, Tskhondiya Nil N and Beklemishev, "Simulating the effect of a high density electric current pulse on the stress field during plastic deformation,” J. ORIGINAL RESEARCH, vol. 5, pp. 157-162, 2012.

[10] Kleemola H.J and Ranta Eskola A J, "Effect of strain rate and deformation temperature on the strain-hardening of sheet steel and brass in uniaxial tension," J. Sheet Metal Industry, vol. 17, pp. 1046-1057, 1979.

[11] Hollomon J H, "Tensile deformation," J. Transactions of AIME, vol. 162, pp. 268-290, 1945. 